\title{
Valorization of Pozzolan Concretes for Thermal Insulation
}

\author{
Eddie Franck Rajaonarison ${ }^{1)}$ \\ 1) Sciences of Materials and Metallurgy, \\ Ecole Supérieure Polytechnique, \\ University of Antananarivo, 101 Antananarivo, \\ Madagascar. \\ Bam Haja Nirina Razafindrabe $e^{3)}$ \\ 3) Faculty of Agriculture, University of the Ryukyus, \\ 1 Senbaru, Nishihara, Okinawa 903-0213, \\ Japan.
}

\begin{abstract}
The present paper deals with the experimental and theoretical studies to demonstrate the potential benefits of pozzolan for thermal insulation purpose. The heat transfer (HT) through a pozzolan concrete partition wall was investigated by focusing on the boundary conditions of entry and exit of the wall as mode of convective and radiative transfers. The various concretes used in this study were made with $5 / 10 \mathrm{~mm}$ and $10 / 20 \mathrm{~mm}$ slag and less than $100 \mu \mathrm{m}$ fines with minimal porosity and appropriate cement dosage. The insulating behavior of concretes was evaluated by means of mathematical relations between different thermophysical parameters and developed on the basis of the finite difference method. The obtained results strongly show the need for optimum mix conditions of pozzolan to produce lightweight concrete.
\end{abstract}

Keywords: Pozzolan; Lightweight concrete; Insulating capacity; Thermophysical properties; Finite differences.

\section{INTRODUCTION}

Lightweight concrete (LWC) has been successfully used since the ancient Roman times. It has continued to gain popularity due to its lower density and superior thermal insulation properties [1]. The light aggregate concrete structure is manufactured with a light aggregate structure as defined in ASTM C330 [2]. All the porous aggregates have their own characteristic properties which in turn affect those of the resulting lightweight concretes. Therefore, the experimental study of the characteristics of the aggregates is of great significance in the process of manufacture of the light concrete in a view to obtaining good thermal performance [3]. Some types of concrete, subject to thermal loads have already been studied [4] [5]. Several researchers have previously worked on lightweight pozzolan-based

\author{
Alexandre Gacoin ${ }^{2)}$ \\ 2) Search Group on Sciences for the Engineers, \\ GRESPI/ Thermomécanique, \\ University of Reims Champagne-Ardennes, \\ Campus du Moulin de la Housse - BP 1039, \\ 51687 Reims Cedex 2, France. \\ Vincent Emile Rasamison ${ }^{4)}$ \\ 4) Researcher at the CNARP Department of Chemistry, \\ B.P.702, 101 Antananarivo, \\ Madagascar.
}

concretes by considering mortar hardness, cement fraction [6], granulate for light concrete [7] [8] and thermal activation [9]. These works have shown that pozzolans are building materials with economic and ecological interests. However, data inferred from experimental and theoretical studies of lightweight concretes are relatively scarce, and are therefore the object of the present report.

The investigation of the thermal behavior of a wall in a habitat can be carried out by using different methods to solve the heat transfer equation, as reported elsewhere [10]. In this research, the finite difference method was performed as it takes into account the resolution of the transient heat transfer equation. The pozzolan concrete wall and its inner face were initially kept at a constant temperature, while an external disturbance was applied on its outer face at a constant temperature. The temperature in the inside was maintained at its initial value. Under these conditions, the main motivations of this work are to produce common light concrete from pozzolan and to disclose its thermal properties and insulating capacity. For these purposes, we evaluated the feasibility and effectiveness of the use of natural aggregates in lightweight concrete from theoretical and practical standpoints.

\section{MATERIALS AND METHODS}

\subsection{Materials}

The following materials were used to produce the various concretes considered in the experimental program.

\subsubsection{Cement}

The cement used during our experiments is of type I (ASTM C150 2009) [11]. Their physical properties and chemical composition are listed in Table 1. 
Table 1: Physical and chemical properties of Cement.

\begin{tabular}{llll}
\hline Specific gravity (g/cm3) & & 3,15 & ASTM C 188-03 \\
Specific surface (cm2/g) & & 3897 & ASTM C 204-05 \\
Setting time initial (min) & & 30 & ASTM C 191-04 \\
Compressive strength (MPa) & & & \\
& $1 \mathrm{~d}$ & 10,4 & \\
& $3 \mathrm{~d}$ & 21,3 & \\
& $7 \mathrm{~d}$ & 33,5 & \\
& $28 \mathrm{~d}$ & 43,6 & \\
Chemical composition, \% by mass & & & \\
& $\mathrm{SiO} 2$ & 20,5 & \\
& $\mathrm{Al2O} 3$ & 4.52 & \\
& $\mathrm{Fe} 2 \mathrm{O} 3$ & 2.71 & \\
& $\mathrm{CaO}$ & 63.93 & \\
& $\mathrm{MgO}$ & 2.39 & \\
& $\mathrm{~K} 2 \mathrm{O}$ & 1.01 & \\
& $\mathrm{SO}$ & 3.3 & \\
& $\mathrm{Na2O}$ & 0.19 & \\
& $\mathrm{LOI}$ & 0.97 & \\
& & & \\
& &
\end{tabular}

\subsubsection{Fine aggregate}

The fines used in some mixtures were $100 \mu \mathrm{m}$ constituents originated from ground pozzolana. Materials smaller than 75 $\mu \mathrm{m}$, equivalent to dust in the slag, were tested according to ASTM C-117 procedure [12]. The proportion of dust generally increases near the soil surface and decreases with depth. The materials taken from the quarry of the main pit are particles with size less than $75 \mu \mathrm{m}$ and a percentage between 0.4 and $0.6 \%$, corresponding to an average of $0.5 \%$. The results indicate that the large scale pozzolan deposition met the requirements of ASTM C-33 [13] for both the row and average proportion of materials thinner than $75 \mu \mathrm{m}$.

\subsubsection{Coarse aggregate}

Locally available pozzolan granulate meets the ASTM C618 classification requirements [14]. The powder was examined using a Siemens D500 diffractometer operating with a monochromatic $\mathrm{CuKa}$ radiation at a wavelength of $\mathrm{k}=$ $1.7903 \mathrm{~A}^{\circ}$, a voltage of $40 \mathrm{kV}$ and a current of $30 \mathrm{~mA}$. The obtained results are shown in Fig.1. Table 2 summarizes the main characteristics of the samples used during this study while Table 3 shows the results of chemical analysis of these samples. With respect to aggregates, their properties were determined according to the standard procedures described in the corresponding ASTM test methods.

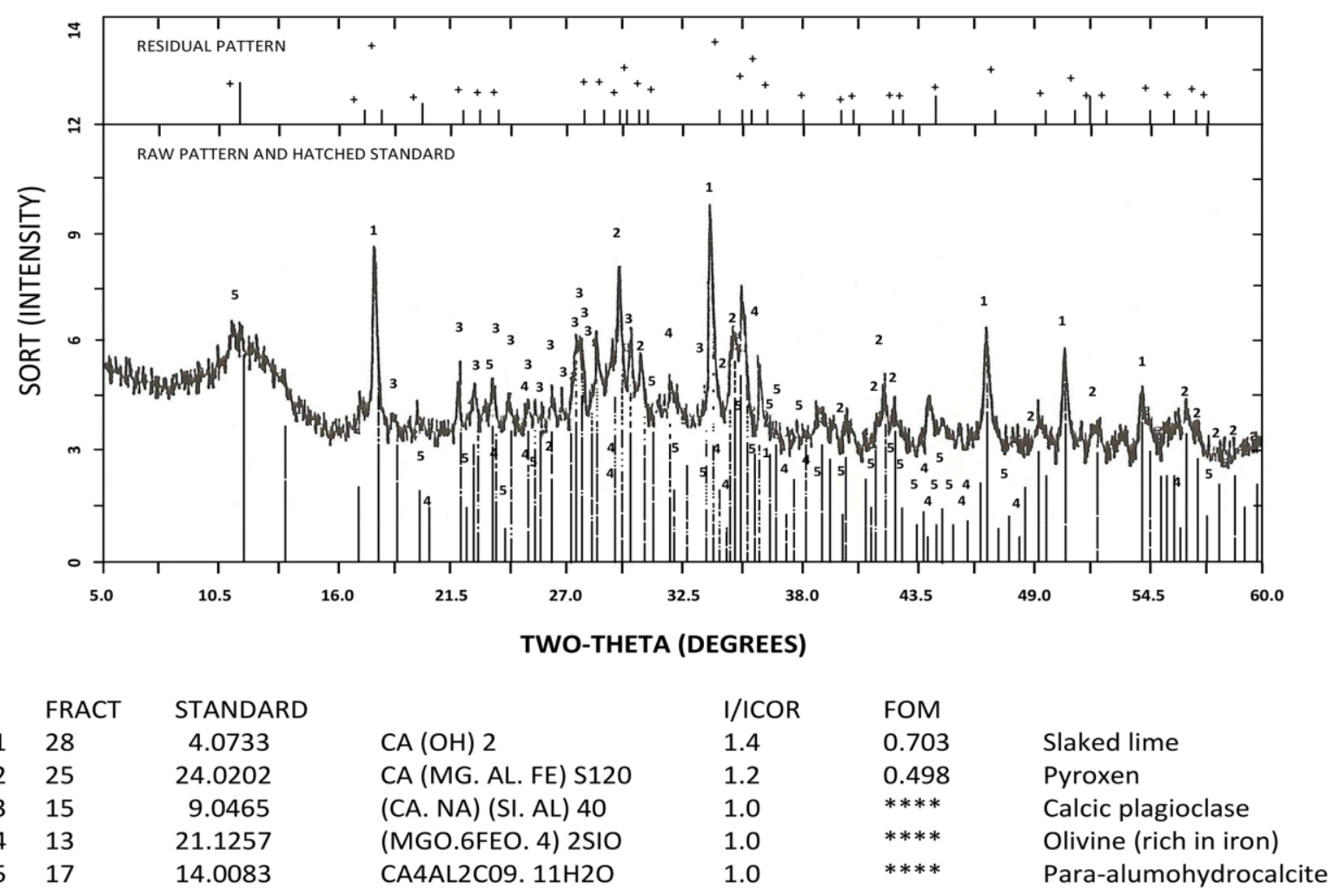

Fig. $1 \mathrm{X}$-ray diffraction analysis. 
Table 2: Pozzolan used in the study.

\begin{tabular}{lllll}
\hline Quarry & Color & Form & State of surface & Structure \\
S1 & purplish-blue black & scoriaceous & Very rough & Ppellation \\
\hline
\end{tabular}

Table 3: Chemical analysis of pozzolans.

\begin{tabular}{ll} 
Elements & \% \\
\hline $\mathrm{SiO}_{2}$ & 48,70 \\
$\mathrm{Al}_{2} \mathrm{O}_{3}$ & 20,12 \\
$\mathrm{Fe}_{2} \mathrm{O}_{3}$ & 01,13 \\
$\mathrm{CaO}$ & 10,58 \\
$\mathrm{MgO}$ & 09,81 \\
$\mathrm{~K}_{2} \mathrm{O}$ & 01,10 \\
$\mathrm{SO}_{3}$ & 00,00 \\
$\mathrm{TiO}_{2}$ & 02,81 \\
$\mathrm{MnO}$ & 00,22 \\
$\mathrm{Na}_{2} \mathrm{O}$ & 02,78 \\
$\mathrm{Cr}_{2} \mathrm{O}_{3}$ & 00,10 \\
$\mathrm{P}_{2} \mathrm{O}_{5}$ & 00,64 \\
$\mathbf{L O I}$ & 02,00 \\
\hline TOTAL & $\mathbf{9 9 , 9 9}$ \\
\hline
\end{tabular}

\subsubsection{Sand}

The siliceous sand used in this study was obtained from the local market. The rolled grains, round shape and smooth surface warrant its quality. The average specific mass is 2.66 $\mathrm{kg} / 1$ for the tested samples. The apparent average density is $1.52 \mathrm{~kg} / 1$ whereas the porosity is $42.86 \%$ and the sand equivalent is $72 \%$.

\subsubsection{Water}

Potable water used meets the requirements of ASTM C160206 [15]. It is employed to mix concrete and treated samples.

\subsection{Test methods}

The production of the concrete mix was carried out as described in ASTM C 192-07 [16]. The proportions were optimized with regards to homogeneity and consistency, and were established for the reference mixtures by test batches for a target slump of $200 \mathrm{~mm} \pm 20 \mathrm{~mm}$. The procedure used to establish the proportions of all mixtures involves many steps. For concrete without sand ie c.1 up to c.6, the cement dosage was gradually increased to meet the results of the vacuum ratio. The initial combination of coarse and fine aggregates was improved to give the minimum void content or the maximum free unit weight. The combination of dough and aggregates was also adapted to obtain an appropriate homogeneity. The actual volume of pozzolan aggregates was kept constant for any compositions, as exemplified by samples from $\mathrm{c} 1$ to $\mathrm{c} 10$. This is to get a maximum effectiveness of vr on the void ratios. The amounts of the fines used were adjusted to respect the total value of the absolute volume of the mixture and in replacement of cement. For blends c. 7 to c.10, ordinary sand was used and the experimental volumetric method was performed according to the ASTM C33 procedure. Table 4 shows the proportions of the mixture for the 10 concrete mixes studied.

Table4: Concretes composition.

\begin{tabular}{lllllllllll}
\hline Dénomination & c.1 & c.2 & c.3 & c.4 & c.5 & c.6 & c.7 & c.8 & c.9 & c.10 \\
\hline $\mathrm{V}_{\mathrm{c}}\left(K g / \mathrm{m}^{3}\right)$ & 85 & 115 & 120 & 120 & 122 & 146 & 81 & 115 & 124 & 147 \\
$\mathrm{~V}_{\mathrm{f}}\left(K g / \mathrm{m}^{3}\right)$ & 00 & 00 & 00 & 77 & 139 & 182 & 00 & 00 & 00 & 50 \\
$\mathrm{~V}_{\mathrm{r}}\left(K g / \mathrm{m}^{3}\right)$ & 537 & 537 & 537 & 537 & 537 & 537 & 537 & 537 & 537 & 537 \\
$\mathrm{Sand}\left(K g / \mathrm{m}^{3}\right)$ & 00 & 00 & 00 & 00 & 00 & 00 & 69 & 105 & 162 & 72 \\
$\mathrm{~W}\left(K g / m^{3}\right)$ & 170 & 170 & 170 & 170 & 170 & 170 & 120 & 160 & 160 & 175 \\
Density $\left(K g / m^{3}\right)$ & 1200 & 1260 & 1265 & 1345 & 1625 & 1715 & 1286 & 1330 & 1400 & 1463 \\
\hline
\end{tabular}




\subsection{Thermal conductivity}

\section{THERMOPHYSICAL CHARACTERIZATION}

It is necessary to quantify the thermal conductivity of any materials designated to play an insulating role by avoiding thermal losses as in many areas including buildings and residential houses. The box method was used to measure thermophysical characteristics. Samples were dried at a temperature of about $50^{\circ} \mathrm{C}$. It was then subjected to an unidirectional current between cold isotherm and the sources of constant heat flux. The thermal gradient that evolves between these two faces was measured when it becomes constant. Once the steady state is established, the apparent thermal conductivity is calculated using the formula [17]:

$$
\lambda a=\frac{e}{S(T c-T f)}\left[\frac{V^{2}}{R}-C_{1}(T b-T a)\right]
$$

Here, $\lambda a$ is the apparent thermal conductivity, $\mathrm{V}$ is the voltage applied to the terminals of the heating plate, $\mathrm{R}$ is the plate resistance, $\mathrm{C}_{1}$ is the heat loss coefficient, Ta is the ambient temperature of the experiment room, Tb is the temperature inside the box, Tc is the temperature of the hot side of the sample, Tf is the temperature of the cold side of the sample, $\mathrm{S}$ is the surface of the sample and e is the sample thickness.

It is known that the thermal conditions of the concrete and its thermophysical properties have a direct influence on its insulation capacity.

\subsection{Thermal diffusivity}

The device used to determine the thermal diffusivity is also the box method as above for the measurement of the thermal conductivity. The sample was placed in a reflective and thermally well insulated box. It receives a thermal pulse from a source of constant flux radiation consisting of a $500 \mathrm{~W}$ incandescent lamp.

\subsection{Massic heat}

The determination of the mass heat of solids can be achieved by several methods, including adiabatic calorimeter, flow calorimeter and falling calorimeter. The choice of a method is essentially guided by the range of temperatures used and the precision sought. Accurate measurements are generally achieved with the adiabatic calorimeter at temperatures ranging from 0 to $500{ }^{\circ} \mathrm{C}$. The specific heat $\mathrm{C}$ is deduced from the relation between the density, conductivity and thermal diffusivity.

$\mathrm{C}=\frac{\lambda}{\rho a}$

where $\mathrm{C}$ is massic heat, $\lambda$ is the thermal conductivity, $\rho$ is the density and a is the thermal diffusivity.

\section{MODELIZATION}

Our main objective is to build a database of time and space steps for meshing for finite difference numerical resolution of the conduction heat transfer equation in the case of a monolayer wall first, and then a bilayer one. This requires the resolution of the one-dimensional heat equation:

$\alpha \frac{\partial^{2} T}{\partial x^{2}}=\frac{\partial T}{\partial t}$

where $\alpha$ is the diffusivity coefficient under the initial condition $T(x, t=0)=T i$ and border conditions. The Fourier condition makes it possible to impose a flow on the entrance face of the wall, as defined by:

- If $\mathrm{x}=0$

$h_{e}\left[T(0, t)-T_{f}(x=0, t)\right]=k_{e} \frac{\partial T(x, t)}{\partial x} \quad x=0$

where $h_{e}$ is the coefficient of convection outside the air, $T(0, t)$ is the temperature on the input side, $T_{f}$ is the outside air temperature and $\mathrm{k}_{\mathrm{e}}$ is the conductivity of the material at the entrance.

$-\quad$ If $\mathrm{x}=\mathrm{e}$
$k_{S}\left(\frac{\partial T(x, t)}{\partial x}\right)_{x=e}=h_{i}\left[T_{i}-T(e, t)\right]$

where $h_{i}$ represents the coefficient of convection inside the house, $T(e, t)$ indicates the temperature on the exit side, $T_{i}$ is the initial temperature of the wall and temperature inside the house, $\mathrm{k}_{\mathrm{s}}$ corresponds to the conductivity of the material at the outlet and $\mathrm{e}$ expresses the wall thickness. 
For the resolution of these equations, it is assumed that the temperature disturbance at the front level does not reach the opposite face. The wall is therefore considered as a semi-infinite solid. This hypothesis remains valid in the case of the multilayer wall. Nevertheless, it must take into account the presence of interface(s). The contact at the interfaces is perfect, leading to the continuity of the temperature. The boundary conditions supplement those of the contact interface where the temperatures of the faces of each medium are the same and there is a conservation of heat flow.

At the interface: $\mathrm{x}=\mathrm{e} 1$

$u_{1}\left(e_{1}, t\right)=u_{2}\left(e_{1}, t\right)$

$k_{1}{\frac{\partial u_{1}(x, t)}{\partial t} \quad x=e_{1}}=k_{2}{\frac{\partial u_{2}(x, t)}{\partial t} \quad x=e_{1}}$

where indices 1 and 2 denote the number of each layer and $k_{i}$ corresponds to the conductivity of the medium $i$.

\subsection{Analytical resolution}

There are different methods which can be put to use for the analytical resolution of the unsteady conduction heat equation. The method using the Laplace transform have been considered as extremely powerful [18]. It is preferable to the method of separating variables when the boundary conditions are time-dependent or when there are no homogeneity. Moreover, this method is very suitable for establishing approximate but rapidly convergent solutions for both small and large values of time from appropriate series developments of the transformed solution. It is known that the series solutions obtained by the method of separating variables converge in general very slowly for the small values of time.

\subsubsection{Case of a single layer wall}

The model adopted in the present study consists of a wall where two outer faces are in contact with atmospheric air and another one with the interior atmosphere of a room. For low values of time, the solution corresponds to that of a semi-infinite wall. It is given by the formula:

$\frac{T-T_{i}}{T_{f}-T_{i}}=\left[\operatorname{erfc}\left(\frac{x}{2 \sqrt{a t}}\right)-\exp \left(\frac{h_{e}}{k} x+\frac{h_{e}^{2}}{k^{2}} a t\right) \operatorname{erfc}\left(\frac{h_{e}}{k} \sqrt{a t}+\frac{x}{2 \sqrt{a t}}\right)\right]$

Where

$\operatorname{erfc}(x)=1-\operatorname{erf}(x)$

and

$\operatorname{erf}(x)=\frac{2}{\sqrt{\Pi}} \int_{0}^{x} \exp \left(-u^{2}\right) d u$

where $\mathrm{x}$ is between 0 and the thickness of the wall.

\subsubsection{Case of a bilayer wall}

Taking into account the change of variable $u=T-T_{i}$ with $T_{i}$ as the initial body temperature, the equations of the continuity of temperature and the conservation of heat flux on the one hand, and the equation systems to be solved on the other hand can be found in Table 5 . The analytical solution formed by $\mathrm{u} 1$ and $\mathrm{u} 2$ is determined by the combinations of several functions erfc.

Table 5: Bilayer wall equation systems.

\begin{tabular}{cc}
\hline 1st "Entry" layer & 2nd layer «exit» \\
\hline$a_{1} \frac{\partial^{2} u_{1}}{\partial^{2} x}=\frac{\partial u_{1}}{\partial t}$ & $a_{2} \frac{\partial^{2} u_{2}}{\partial^{2} x}=\frac{\partial u_{2}}{\partial t}$ \\
$\lim _{x \rightarrow \infty} u_{2}(x, t)=0$ \\
$-k_{1} \frac{\partial u_{1}(x, t)}{\partial x}{ }_{x=0}=h_{e}\left[u_{f}-u_{1}(0, t)\right]$ & and \\
$u_{1}\left(e_{1}, t\right)=u_{2}\left(e_{1}, t\right)$ & $k_{1} \frac{\partial u_{1}(x, t)}{\partial x}{ }_{x=e_{1}}=k_{2} \frac{\partial u_{1}(x, t)}{\partial x}{ }_{x=e_{2}}$ \\
\hline
\end{tabular}

\subsection{Numerical resolution by finite difference method}

In a mesh defined in the plane of the space and time variables $(x, t)$, the nodes are identified using the indices $i$ and $n$ corresponding to the discrete value of the temperature at the node $(\mathrm{i}, \mathrm{n})(\mathrm{x}=\mathrm{i} \Delta \mathrm{x}, \mathrm{t}=\mathrm{n} \Delta \mathrm{t})$. There are three types of models: the implicit model; the Crank-Nicholson scheme and the explicit model. According to Lax's equivalence theorem, "a linear and consistent finite difference scheme is convergent if and only if it is stable". The Cranck-Nicholson scheme has the double merit of being unconditionally stable and leading to the lowest discretization errors [19]. The implicit model may present instability. [20] Explicit and implicit finite difference schemes can be combined and described by: 
$\frac{T_{i}^{k+1}-T_{i}^{k}}{\Delta t}=a\left[\sigma \frac{T_{i+1}^{k+1}-2 T_{i}^{k+1}+T_{i-1}^{k+1}}{(\Delta x)^{2}}+(1-\sigma) \frac{T_{i+1}^{k}-2 T_{i}^{k}+T_{i-1}^{k}}{(\Delta x)^{2}}\right]$

If $\sigma=0$ and $\sigma=1$, we obtain a system of explicit finite difference equations and a system of implicit equations, respectively.

The heat transfer discretization equation in the Crank-Nicholson scheme is formulated using the following relationship by always adopting the variable change $\mathrm{u}=\mathrm{T}-\mathrm{T}_{\mathrm{i}}$ :

$u_{i-1}^{j+1}-2\left(1+\frac{1}{M}\right) u_{i}^{j+1}+u_{i+1}^{j+1}=-u_{i-1}^{j}+2\left(1-\frac{1}{M}\right) u_{i}^{j}-u_{i+1}^{j}$

where $\mathrm{j}$ corresponds to $\mathrm{t}$ and $\mathrm{j}+1$ to $\mathrm{t}+\mathrm{dt}$

This relation shows link between the discrete temperatures and the nodes $i,(i+1)$, and $(i-1)$ computed at time $j$ and at the temperatures of the same nodes at time $(j+1)$. In this equation, $M$ is a dimensionless parameter which is characteristic of the finite difference scheme and is given by the expression:

$M=a \frac{\Delta t}{\Delta x^{2}}$

The node on the input face is assigned the index $0(\mathrm{i}=0)$ and the next node 1 . At the input face, the transfer equation according to the Cranck-Nicholson scheme is described by:

$u_{1}^{j+1}-\left(1+\frac{1}{M}+\frac{h_{e}}{k} \Delta x\right) u_{0}^{j+1}=-u_{1}^{j}+\left(1-\frac{1}{M}+\frac{h_{e}}{k} \Delta x\right) u_{0}^{j}-2 \frac{h_{e}}{k} \Delta x * u_{f}$

The choice of mesh method is mainly based on the comparison between the analytical solution of a simple problem of the semiinfinite wall with the corresponding solution given by the Cranck-Nicholson scheme.

\subsubsection{Case of the single layer wall}

To be identical to the solution given by the Cranck-Nicholson scheme, the analytical solution has to satisfy the equality, as defined by:

$$
\frac{2 \Delta x \frac{h_{e}}{k}}{1+\frac{h_{e}}{k} \Delta x+\frac{\Delta x^{2}}{a \Delta t}}=\left[1-\exp \left(\frac{h_{e}^{2}}{k^{2}} a \Delta t\right) \operatorname{erfc}\left(\frac{h_{e}}{k} \sqrt{a \Delta t}\right)\right]
$$

This equation contains two unknowns, namely $\Delta \mathrm{x}$ and $\Delta \mathrm{t}$. It can be solved only if there is a relationship between both of them. By asking:

$y_{1}=\frac{2 \Delta x \frac{h_{e}}{k}}{1+\frac{h_{e}}{k} \Delta x+\frac{\Delta x^{2}}{a \Delta t}}$ of graph (c1) and

$y_{2}=\left[1-\exp \left(\frac{h_{e}^{2}}{k^{2}} a \Delta t\right) \operatorname{erfc}\left(\frac{h_{e}}{k} \sqrt{a \Delta t}\right)\right]$ of graph (c2)

The representative curves are in Fig. 2.

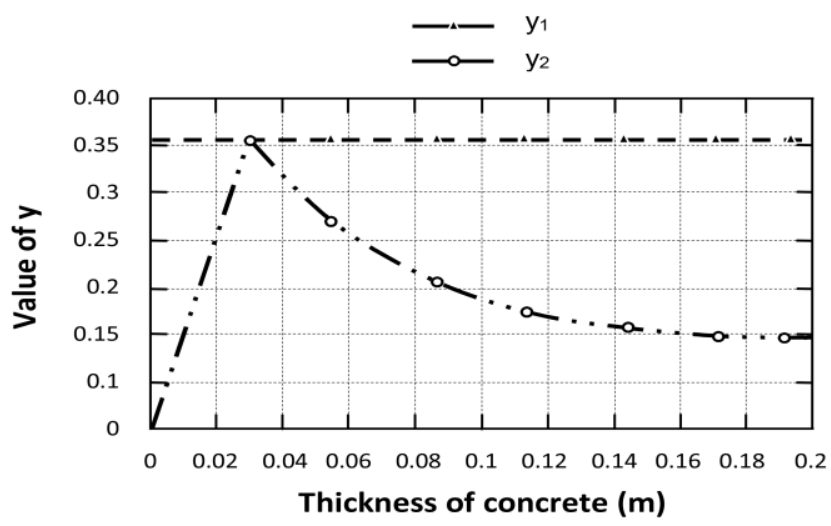

Fig. 2 Curve shape y1 and y2.

The function y1 goes through a maximum for $\Delta x=\sqrt{a \Delta t}$. At the point of tangency, y1 $\max =\mathrm{y} 2$.

$\frac{2 \frac{h_{e}}{k} \sqrt{a \Delta t}}{2+\frac{h_{e}}{k} \sqrt{a \Delta t}}=\left[1-\exp \left(\frac{h_{e}}{k} \sqrt{a \Delta t}\right)^{2} \operatorname{erfc}\left(\frac{h_{e}}{k} \sqrt{a \Delta t}\right)\right]$

For the solution of $\Delta \mathrm{t}$, draw the $\mathrm{z} 1$ and $\mathrm{z} 2$ functions by asking:

$x=\frac{h_{e}}{k} \sqrt{a \Delta t}$

$z_{1}(x)=\frac{2 x}{2+x}$ and

$z_{2}(x)=1-\exp \left(x^{2}\right) \operatorname{erfc}(x)$ to see the intersection of these curves in fig. 3 . 


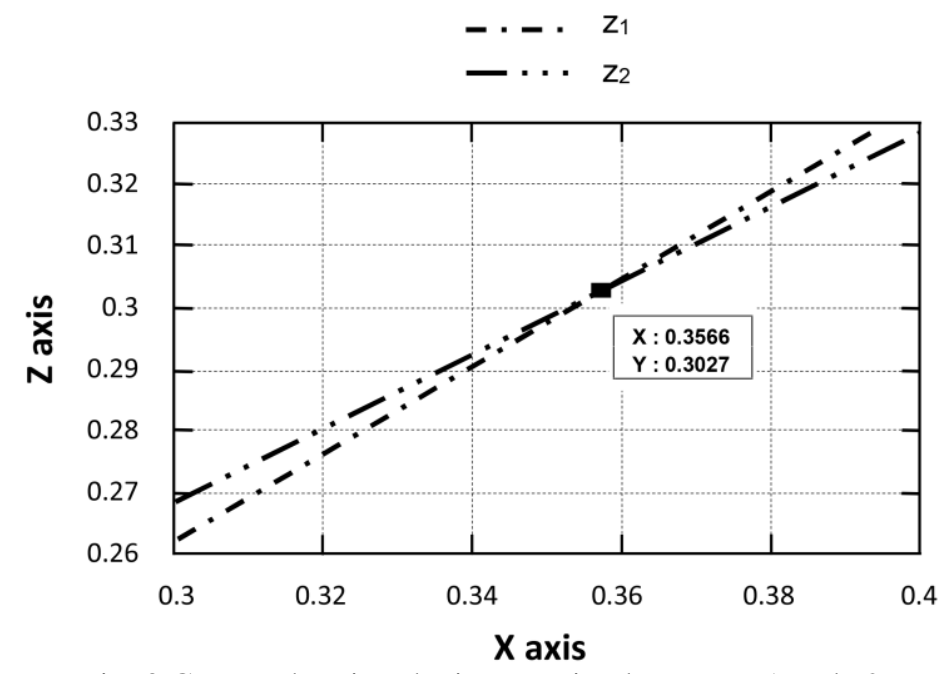

Fig. 3 Curves showing the intersection between $\mathrm{z} 1$ and $\mathrm{z} 2$.

From the graphs of $\mathrm{z} 1$ and $\mathrm{z} 2$, the equality (A) is checked for $\frac{h_{e}}{k} \sqrt{a \Delta t} \approx 0.357$

The time step is thus determined by the formula:

$\Delta t=\left(0.36 \frac{k}{h_{e} \sqrt{a}}\right)^{2}$ ou $\Delta t=\left(0.36 \frac{b}{h_{e}}\right)^{2}$

where $b$ is the thermal effusivity of the material, a is its diffusivity, $k$ is its conductivity and $h_{e}$ is the external convection coefficient. We can deduce $\Delta x$ as follow:

$\Delta x=0,36 \frac{k}{h_{e}}$

The characteristic parameter $\mathrm{M}$ of the finite difference scheme is therefore determined as:

$M=a \frac{\Delta t}{\Delta x^{2}}=1$

\subsubsection{Multilayer wall case}

The discretization of the heat flow conservation equation using the Cranck-Nicholson scheme in finite difference method is described below. The method consists in extending a fictional mesh in each medium into another medium. First, one needs to draw the fictional mesh in each medium and then extend it in each side.

$-u_{i \max -1}^{j}+u_{\text {imax }}^{j}\left[\left(1-\frac{1}{M_{k}}\right)+K_{a}\left(1+\frac{1}{M_{m}}\right)\right]-K_{a} u_{\text {imax }-1}^{j}=u_{i \max -1}^{j+1}-u_{i \max }^{j+1}\left[\left(1+\frac{1}{M_{k}}\right)+K_{a}\left(1+\frac{1}{M_{m}}\right)\right]+K_{a} u_{i \max +1}^{j+1}$

with $K_{a}=\frac{K_{m} \Delta x_{k}}{K_{k} \Delta x_{m}} ; M_{m}=\frac{a_{m} \Delta t}{\Delta x_{m}^{2}} ; M_{k}=\frac{a_{k} \Delta t}{\Delta x_{k}{ }^{2}}$;

where $\mathrm{k}_{\mathrm{i}}$ is the conductivity of medium $\mathrm{I}, \Delta \mathrm{x}_{\mathrm{i}}$ represents the space step of medium $\mathrm{I}, \mathrm{a}_{\mathrm{i}}$ represents the diffusivity of medium I and $\Delta \mathrm{t}$ corresponds to the time step.

\subsection{Application to pozzolan concrete}

The wall is composed of different materials including cements and pozzolans with or without river sand. Monolayer walls are first and foremost unsprayed concrete with or without sand, ie concrete c1 to c3 and c7 to c9, then other concretes, ie $\mathrm{c} 4$ to $\mathrm{c} 6$ concretes and $\mathrm{c} 10$. The porosity of the materials decreases as the density increases. For each material, a single moisture level called "useful moisture content" is conventionally allowed [21]. It is determined by drying to a constant mass in a controlled oven at $70^{\circ} \mathrm{C}$ and vented with air in a controlled atmosphere at $20^{\circ} \mathrm{C}$ and $65 \%$ relative humidity. The solid heat of the solid, for a constant temperature equal to $20^{\circ} \mathrm{C}$. and corresponding to a useful moisture content of $0.5 \%$, is given by:

$C_{p}=0,84 \mathrm{~kJ} \mathrm{~kg}^{-1} \mathrm{~K}^{-1}$

\subsubsection{Water}

In the case of saturated material, at the temperature of $20^{\circ} \mathrm{C}$, the corresponding specific gravity and the specific heat of the water at constant pressure are:

$\rho_{e} \approx 1000 \mathrm{kgm}^{-3} ; C_{p e}=4183 \mathrm{Jkg}^{-1} \mathrm{~K}^{-1}$

\subsubsection{Air}

In the case of air, at the temperature of $20^{\circ} \mathrm{C}$ and atmospheric pressure, these constants are:

$\rho_{a}=1,205 \mathrm{kgm}^{-3} ; C_{p a}=1005 \mathrm{Jkg}^{-1} \mathrm{~K}^{-1}$

Knowing the low density of the air, its heat density is no consideration. 


\subsubsection{Coefficient of transfer by external convection}

The outdoor convection coefficient varies according to the wind speed with an average of $8 \mathrm{kmh}-1$, ie: $2.2 \mathrm{~ms}-1$. According to the Sturrock model [22][23][24], the respective values of $h_{c s}$ in the lee side and $h_{c a}$ in the weather side are 5.7 V and 11.4+ 4.7 V, where $\mathrm{V}$ is the wind speed $(\mathrm{m} / \mathrm{s})$.

Applying the formulas given for the Sturrock model, the wind and leeward coefficients of wind and lee are 21.8 and $12.7 \mathrm{Wm}$ $2 \mathrm{~K}-1$, respectively.

\subsubsection{Geometric dimensions of the wall}

The wall is characterized by the following geometric dimensions:

- Thickness of concretes $\mathrm{c} 1$ to $\mathrm{c} 3$ and $\mathrm{c} 7$ to $\mathrm{c} 9: \mathrm{e}=11 \mathrm{~cm}$

- Thickness of concrete $\mathrm{c} 4$ to $\mathrm{c} 6 \mathrm{e}=22 \mathrm{~cm}$

- Thickness of concrete $\mathrm{c} 10$ : $\mathrm{e}=5 \mathrm{~cm}$

- Thickness of marble: $\mathrm{e}=5 \mathrm{~cm}$.

\subsubsection{Temperatures}

The value of the initial temperature is the internal temperature at $\mathrm{Ti}=20^{\circ} \mathrm{C}$. The maximum daily temperature is $30^{\circ} \mathrm{C}$. It is also the temperature causing the outside disturbance. This research was conducted under the conditions of the temperatures ranging between 20 and $30^{\circ} \mathrm{C}$, which are generally observed in many parts of Madagascar.

\section{RESULTS AND DISCUSSION}

\subsection{Results from the Implicit Schema Application.}

The following graph shows an example of a result of an implicit schema in the unstable case.

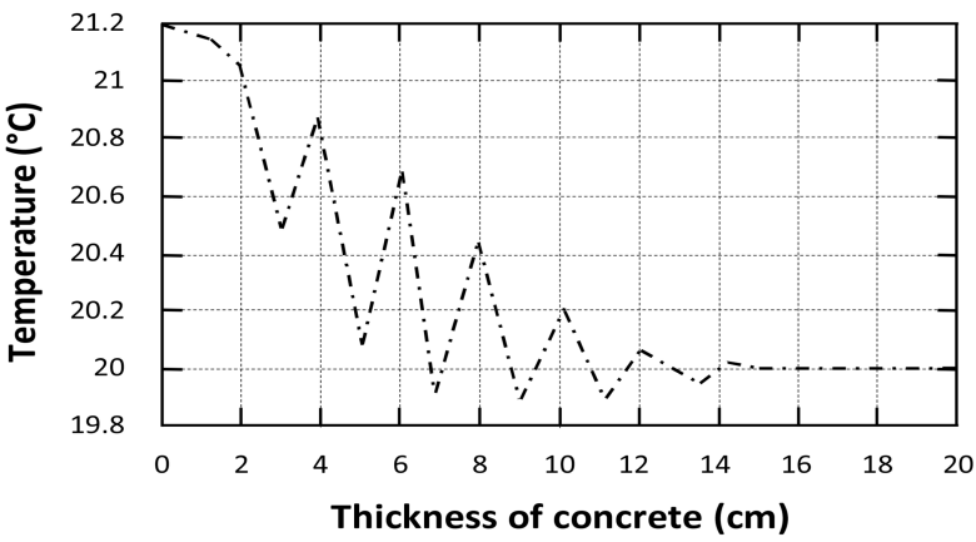

Fig. 4 Behavior of curve from implicit schema of finite differences in unstable case.

Fig. 4 shows curve obtained for a pozzolan concrete wall whose thermal characteristics are as follows:

- Coefficient of conduction: $\mathrm{k}=1,54 \mathrm{Wm} 1 \mathrm{~K}-1$

- Volume heat: $\rho \mathrm{Cp}=1197103 \mathrm{Jm}-3 \mathrm{~K}-1$

The external convection coefficient for the wind wall case was also calculated:

- Coefficient of external convection: hca=21,8 Wm$2 \mathrm{~K}-1$

- Initial and interior temperatures of the wall: $\mathrm{T}_{\mathrm{i}}=$ $20^{\circ} \mathrm{C}$

- Disturbance temperature at the entrance: $\mathrm{T}_{\mathrm{f}}=30^{\circ} \mathrm{C}$

- Dimensionless parameter $\mathrm{M}=0,55$

The choice of the dimensionless parameter $\mathrm{M}$ is linked to the schema. Unfortunately, due to the wrong choice, there are anomalies which lead to the instability of the result.

\subsection{Results from Cranck-Nicholson diagram for single layer} walls.

More than sixty curves were recorded during our experiment. In this paper, only few samplings chosen arbitrarily are shown. Two different curves, shown in fig.6, fig. 7 and fig. 8 , according to the disposition of the wall with respect to the direction of the wind are presented. One is associated with the distribution of the temperature obtained using the analytical solution and the finite difference method by adopting the Crank Nicholson scheme for a fixed time, and the other is related to the percentage difference between the analytical solution and the numerical solution for the same fixed time. This time value is taken as the minimum time at which the percentage of maximum gap between analytical solution and digital solution at the input face is equal to $5 \%$. The input face corresponds to the abscissa axis $\mathrm{x}=0 \mathrm{~m}$.

When a time value greater than the minimum time indicated is considered, the numerical solution (NS) obtained from the use of meshes is very close to the analytical solution (AS). In the case of walls with a single layer, facing the wind, composed of concretes $\mathrm{c} 5$, the results obtained are presented in fig. 5. 


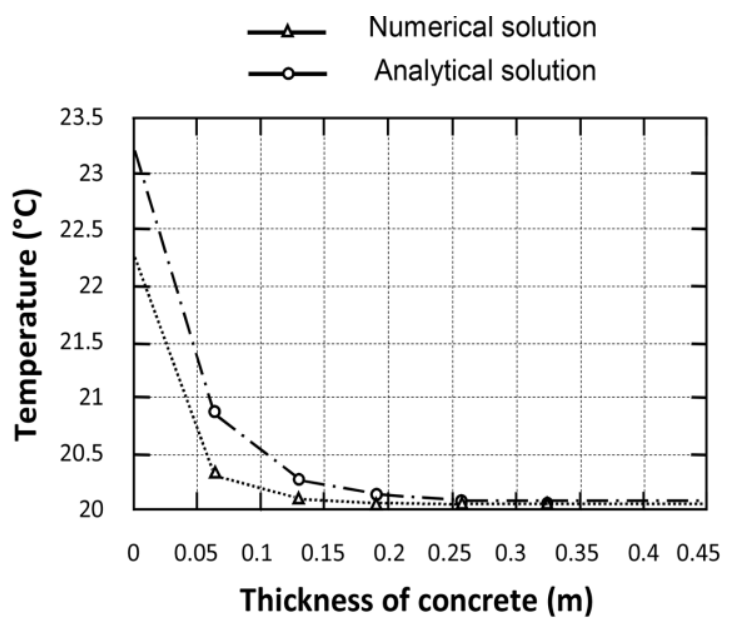

(a)

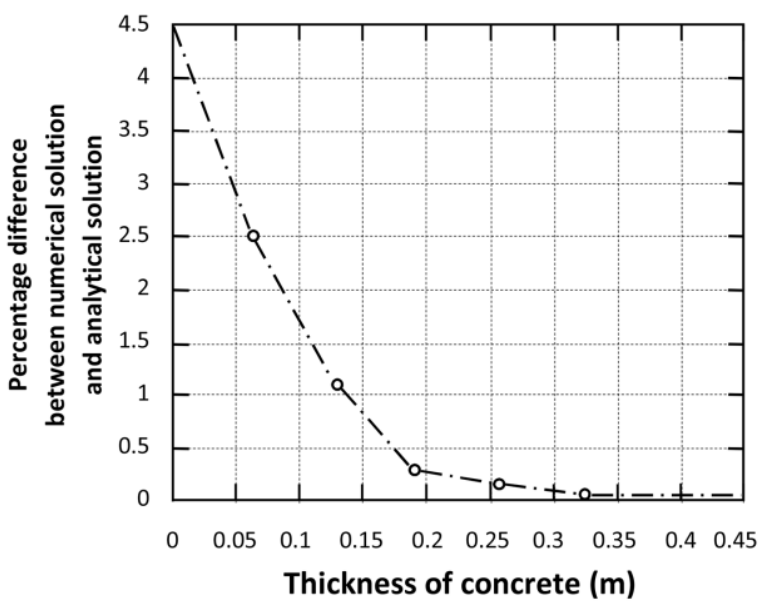

(b)

Fig. 5 (HT) in the Concretes c5; (a) Solutions for $\mathrm{t}=1000 \mathrm{~s}$; (b) Percentage difference between (AS) and (NS) for $\mathrm{t}=1000 \mathrm{~s}$.

The respective results of concretes $\mathrm{c} 4, \mathrm{c} 5$ and c6 represent substantially the same value. That of c5 is more representative, in terms of a mean value, of these three concretes.

The minimum time is the time from which the temperature difference given by the analytical solution versus the digital solution at the input face is less than $5 \%$.

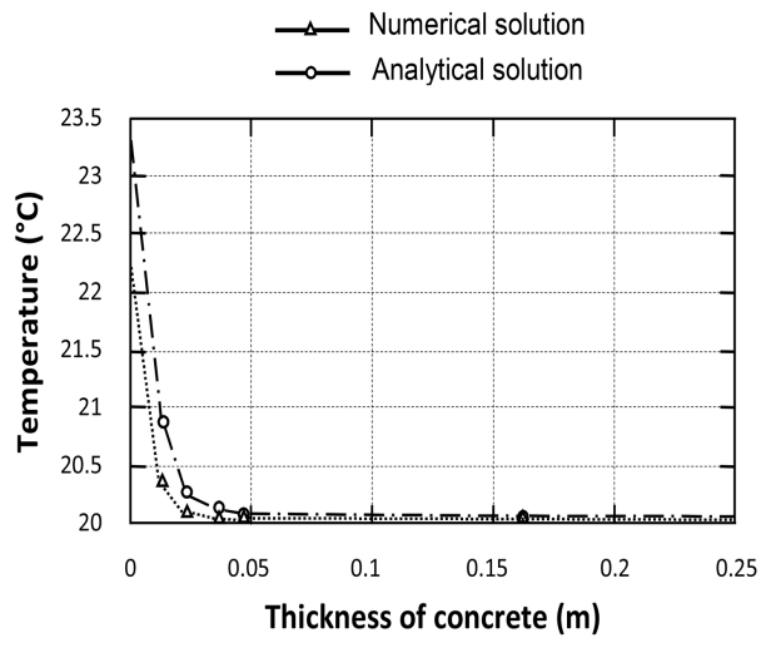

(a)
Two states were considered for the wall composed of concretes $\mathrm{c} 1$ and $\mathrm{c} 7$. The first state, wall to the wind, shown in fig. 6 is the wet one corresponding to the percentage of water in the pores equal to $0.5 \%$, which is the useful moisture content. The second one, lee wall, shown in fig. 7 corresponds to the wall in the saturated condition. In each state, we take into account the direction of the wind in relation to the position of the wall.

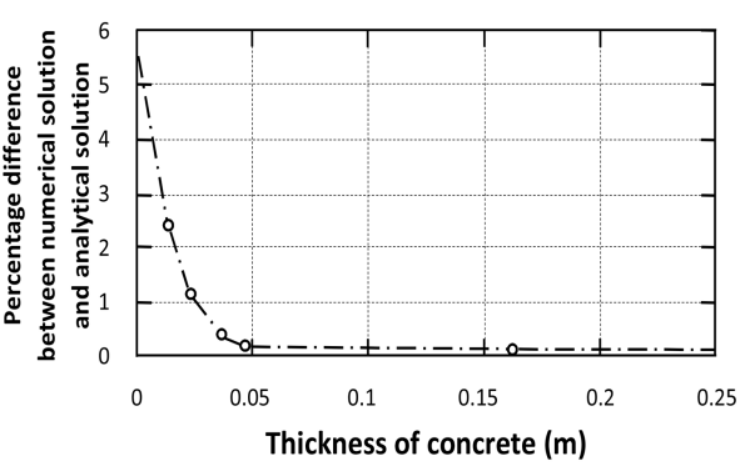

(b)

Fig. 6 (HT) in the concretes c3; (a) Solutions for $\mathrm{t}=100 \mathrm{~s}$. (b) Percentage difference between (AS) and (NS) for $\mathrm{t}=100 \mathrm{~s}$. 


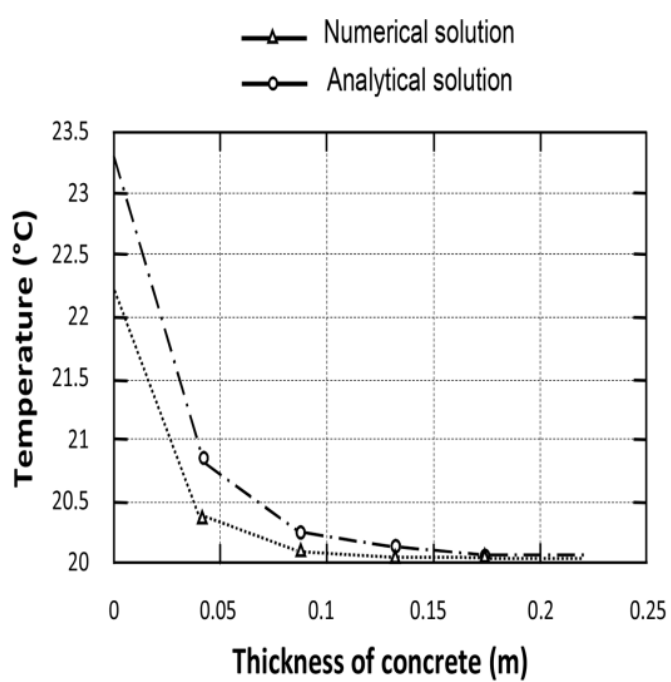

(a)

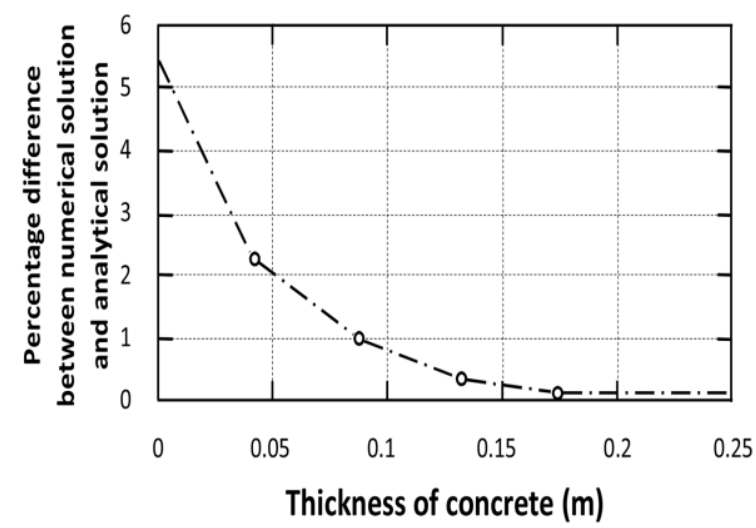

(b)

Fig. 7 (HT) in the concretes c8; (a) Solutions for $\mathrm{t}=1800 \mathrm{~s}$. (b) Percentage difference between (AS) and (NS) for $\mathrm{t}=1800 \mathrm{~s}$.

The average values of the heat transfer results, in concretes $\mathrm{c} 1$ and $\mathrm{c} 2$, are close to that of $\mathrm{c} 3$. Similarly, those obtained with concretes $\mathrm{c} 7$ and $\mathrm{c} 9$ are tangent to the result of $\mathrm{c} 8$.

The minimum time is the time from which the temperature difference given by the analytical solution versus the digital solution at the input face is less than $5 \%$. The overall behavior of the curves indicates that the characteristic parameters of the mesh increase with decreasing value of the convection coefficient. The data also suggest that the wetter the material is, the higher is the minimum time from which the temperature difference given by the digital solution at the input side, and the higher are the characteristic parameters of the mesh.

As far as the time step $\Delta \mathrm{t}$ and the space step $\Delta \mathrm{x}$ are concerned, they decrease with increasing external convection coefficient $\mathrm{h}$. It is worth mentioning that the conductive material is effusive, and the meshing parameters increase with increasing conductivity and effusivity of the material.

Our results were compared with literature data concerning other materials which are supposed to exhibit insulating capacity as shown in Table 6.

Table 6: Synthesis of results in the case of single layer wall.

\begin{tabular}{|c|c|c|c|c|c|c|}
\hline \multirow[t]{2}{*}{ Materials } & \multirow{2}{*}{$\begin{array}{c}\text { Diffusivity } \\
\left(\mathrm{m}^{2} / \mathbf{s}\right)\end{array}$} & \multirow{2}{*}{$\begin{array}{c}\text { Effusivity } \\
\left(\mathbf{J s}^{-1 / 2} \mathbf{m}^{-2} \mathbf{K}^{-1}\right)\end{array}$} & \multirow{2}{*}{ 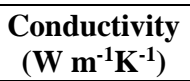 } & \multirow{2}{*}{$\begin{array}{c}\mathbf{h}_{\mathbf{e}} \\
\left(\mathbf{W} \mathbf{m}^{-2} \mathbf{K}^{-1}\right)\end{array}$} & \multicolumn{2}{|c|}{ Maillages meshes } \\
\hline & & & & & $\Delta t(s)$ & $\Delta \mathbf{x}(\mathbf{m})$ \\
\hline \multirow{4}{*}{ Marble } & & & & 10 & 8300 & $10^{-1}$ \\
\hline & $1,2810^{-6}$ & 2600 & 2,95 & 14,3 & 4050 & $710^{-2}$ \\
\hline & & & & 26 & 1225 & $410^{-2}$ \\
\hline & & & & 10 & 2,5 & $1,510^{-3}$ \\
\hline \multirow[t]{3}{*}{ polystyrene } & $810^{-7}$ & 45 & 0,04 & 14,3 & 1,2 & $10^{-3}$ \\
\hline & & & & 26 & 0,4 & $510^{-4}$ \\
\hline & & & & 12,7 & 5909 & $9,810^{-2}$ \\
\hline Granitic rock & $1,6410^{-6}$ & 2700 & 3,5 & 21,8 & 2005 & $5,710^{-2}$ \\
\hline c3 with a moisture & & & & 12,7 & 619 & $1,810^{-2}$ \\
\hline content of $0.5 \%$ & $5,410^{-7}$ & 882 & 0,65 & 21,8 & 210 & $1,110^{-2}$ \\
\hline c3 in the state & & & & 12,7 & 3752 & $4,310^{-2}$ \\
\hline Saturated & $5,010^{-7}$ & 2185 & 1,54 & 21,8 & 1273 & $2,510^{-2}$ \\
\hline c5 with a moisture & & & & 12,7 & 760 & $2,010^{-2}$ \\
\hline content of $0.5 \%$ & $5,110^{-7}$ & 977 & 0.70 & 21,8 & 258 & $1,110^{-2}$ \\
\hline c5 in the state & & & & 12,7 & 3988 & $4,810^{-2}$ \\
\hline Saturated & $5,810^{-7}$ & 2240 & 1,71 & 21,8 & 1353 & $2,810^{-2}$ \\
\hline c8 with a moisture & & & & 12,7 & 1039 & $2,410^{-2}$ \\
\hline content of $0.5 \%$ & $5,510^{-7}$ & 1141 & 0,85 & 21,8 & 353 & $1,410^{-2}$ \\
\hline c8 in the state & & & & 12,7 & 4078 & $5,510^{-2}$ \\
\hline Saturated & $7,310^{-7}$ & 2264 & 1,94 & 21,8 & 1384 & $3,210^{-2}$ \\
\hline
\end{tabular}


The calculated values for the convection coefficient are around $12 \mathrm{Wm}-2 \mathrm{~K}-1$ for a wall downwind and around 20 $\mathrm{Wm}-2 \mathrm{~K}-1$ for a wall exposed to the wind. Granitic rock is slightly more conductive and effusive than marble. The moisture content in pozzolan concrete has an influence on its effusiveness and conductivity. With respect to concrete, the saturated lightweight pozzolan concrete is more conductive and effusive than pozzolan concrete with a moisture content of $0.5 \%$. Compared to polystyrene, these different materials are current drivers. The following materials are arranged according to these insulation capacities: polystyrene, pozzolan concrete with a moisture content of $0.5 \%$, concrete, pozzolan concrete in the state saturated, marble and masonry rubble stone granite.

\subsection{Wall bilayer.}

In this experiment, we will consider a wall in $\mathrm{c} 1$ to $\mathrm{c} 3$ and $\mathrm{c} 7$ to $\mathrm{c} 9: \mathrm{e}=11 \mathrm{~cm}$ whose moisture content is equal to $0.5 \%$

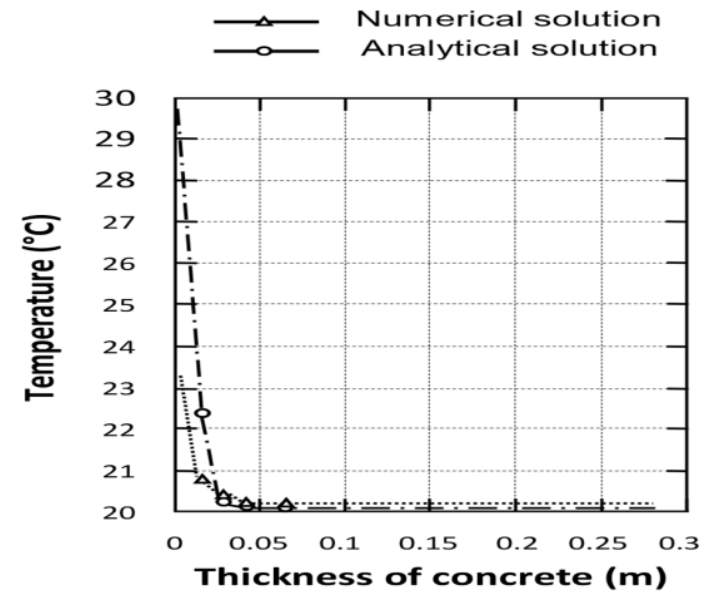

(a) coupled with a layer of $\mathrm{c} 10$ or marble: $\mathrm{e}=5 \mathrm{~cm}$ inside. However, suitable results cannot be obtained since the pitch of the calculated space is greater than the thickness of the wall. The space step is $\Delta \mathrm{x}=4.8 \mathrm{~cm}$ whereas the calculated thickness of $\mathrm{c} 10$ is equal to $5 \mathrm{~cm}$. Two different curves according to the disposition of the wall with respect to the direction of the wind are presented herein. The first curve shows the distribution of the temperature obtained using the analytical solution and using the finite difference method by opting for the Crank Nicholson scheme for a fixed time. The second one gives the percentage difference between the analytical solution and the numerical solution for the same fixed time. Some examples of these curves are illustrated below.

In the case of bilayer wall facing wind, composed of c3 plus $\mathrm{c} 10$, the results obtained are shown in fig. 8 . Still, in the case of Wall under the wind, Fig. 9 gives the solutions of the Wall composed of $\mathrm{c} 8$ plus $\mathrm{c} 10$.

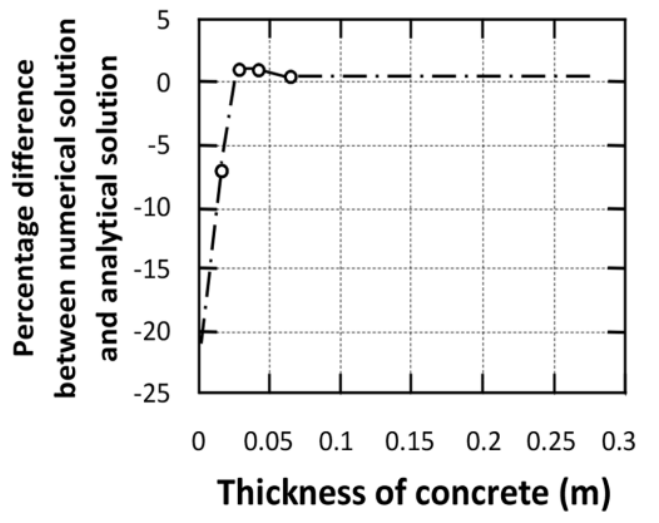

(b)

Fig. $8(\mathrm{HT})$ in the concretes $\mathrm{c} 3+\mathrm{c} 10$; (a) Solutions for $\mathrm{t}=100 \mathrm{~s}$; (b) Percentage difference between (AS) and (NS) for $\mathrm{t}=100 \mathrm{~s}$.

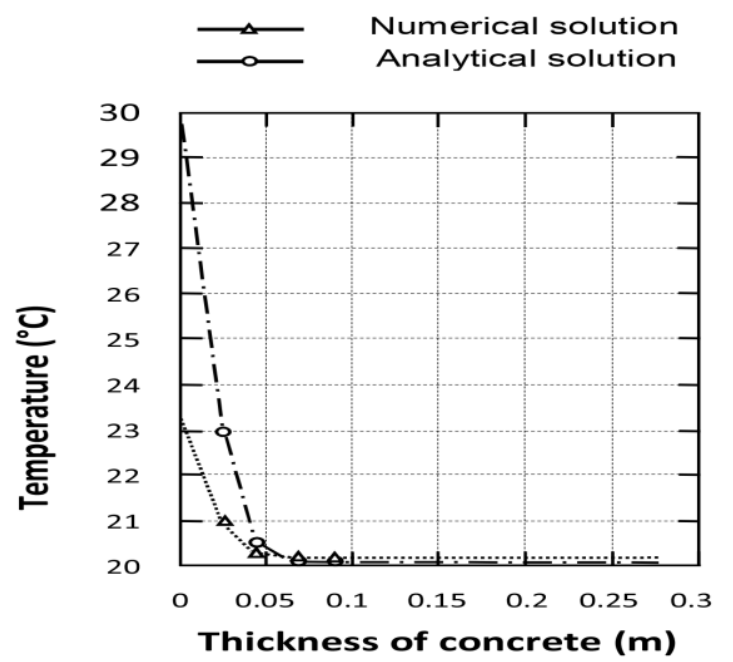

(a)

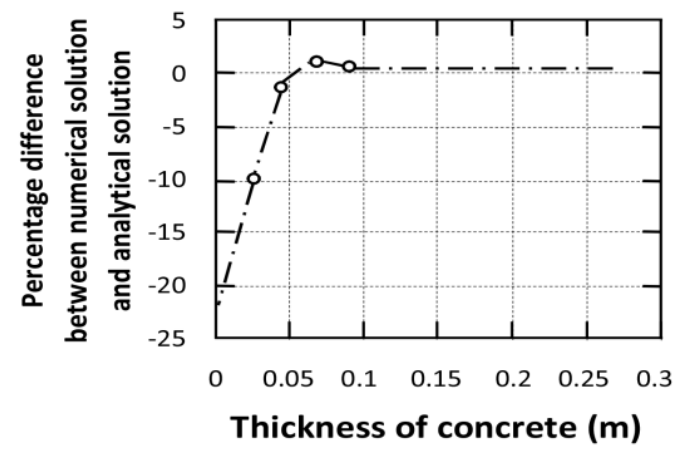

(b)

Fig. $9(\mathrm{HT})$ in the concretes $\mathrm{c} 8+\mathrm{c} 10$; (a) Solutions for $\mathrm{t}=500 \mathrm{~s}$; (b) Percentage difference between (AS) and (NS) for $\mathrm{t}=500 \mathrm{~s}$. 
The input face corresponds to the abscissa axis $\mathrm{x}=0 \mathrm{~m}$. With this value, we have noted a fairly high maximum difference of $22 \%$ at the input face whatever the chosen time value.

There are some cases where the time step and space step values do not lead to acceptable estimates. This is due to the fact that on the one hand, the space step found is greater than the material thickness in practical use and, on the other hand, the time step is not small enough from our observations.
The effectiveness of the method is linked to the distance of the meshes, the thickness of the material and the observation time of the phenomenon. The reliability of the result of the method depends on the number of stitches for the stitches. c10 concrete is more insulating than marble. Let's use the latter to determine the characteristics of the bilayer wall of some pozzolan concretes. The results are given in Table 7 and Table 8 .

Table 7: Characteristic parameters for pozzolan concrete plus marble.

\begin{tabular}{|c|c|c|c|c|c|c|c|c|c|}
\hline \multirow{2}{*}{$\begin{array}{l}\text { Concrete } \\
\text { of the } \\
\text { layer }\end{array}$} & \multirow{2}{*}{$\begin{array}{l}\text { type } \\
\text { first }\end{array}$} & \multirow{2}{*}{$\begin{array}{l}\text { Percentage of water in } \\
\text { the pores } \\
(\%)\end{array}$} & \multirow[t]{2}{*}{$\begin{array}{l}\text { Position relative to } \\
\text { the wind direction }\end{array}$} & \multicolumn{2}{|c|}{$\begin{array}{l}\text { Conductivity } \\
\left(\mathbf{W m}^{-1} \mathbf{K}^{-1}\right)\end{array}$} & \multicolumn{2}{|c|}{$\begin{array}{l}\text { Diffusivity a } \\
\left(\mathbf{m}^{2} \mathbf{s}^{-1}\right)\end{array}$} & \multicolumn{2}{|c|}{$\begin{array}{l}\text { Effusivity b } \\
\left(\mathbf{J s}^{-0.5} \mathbf{m}^{-2} \mathbf{K}^{-1}\right)\end{array}$} \\
\hline & & & & $\begin{array}{l}1 \mathrm{st} \\
\text { layer }\end{array}$ & $\begin{array}{l}2 \mathrm{nd} \\
\text { layer }\end{array}$ & 1st layer & 2nd layer & $\begin{array}{l}1 \mathrm{st} \\
\text { layer }\end{array}$ & $\begin{array}{l}\text { 2nd } \\
\text { layer }\end{array}$ \\
\hline c3 & & 0,5 & $\begin{array}{l}\text { windward } \\
\text { leeward }\end{array}$ & 0,65 & 2,95 & $5,410^{-7}$ & $1,2810^{-6}$ & 882 & 2600 \\
\hline \multirow[t]{2}{*}{$\mathrm{c} 5$} & & 0,5 & windward & & & & & & \\
\hline & & 0,5 & $\begin{array}{l}\text { leeward } \\
\text { windward }\end{array}$ & 0,70 & 2,95 & $5,110^{-7}$ & $1,2810^{-6}$ & 977 & 2600 \\
\hline c8 & & & leeward & 0,85 & 2,95 & $5,510^{-7}$ & $1,2810^{-6}$ & 1141 & 2600 \\
\hline
\end{tabular}

Table 8: Result of meshes for pozzolan concrete plus marble.

\begin{tabular}{|c|c|c|c|c|c|}
\hline $\begin{array}{l}\text { Concrete type } \\
\text { of the first } \\
\text { layer }\end{array}$ & $\begin{array}{c}\text { Percentage } \\
(\%)\end{array}$ & $\begin{array}{l}\text { Position relative to the } \\
\text { wind direction }\end{array}$ & $\begin{array}{c}\text { Coefficient } \\
\text { convection } h\left(\mathrm{Wm}^{-}\right. \\
\left.{ }^{2} \mathrm{~K}^{-1}\right)\end{array}$ & Time pace $\Delta t(s)$ & $\begin{array}{c}\text { Space pace } \Delta \mathbf{x}_{1} \\
(\mathrm{~m})\end{array}$ \\
\hline \multirow[t]{2}{*}{ c3 } & 0,5 & windward & 21,8 & 1976 & $3,610^{-2}$ \\
\hline & & leeward & 12,7 & 4366 & $4,610^{-2}$ \\
\hline \multirow[t]{2}{*}{$\mathrm{c} 5$} & 0,5 & windward & 21,8 & 2426 & $3,910^{-2}$ \\
\hline & & leeward & 12,7 & 5361 & $5,010^{-2}$ \\
\hline \multirow[t]{2}{*}{ c8 } & 0,5 & windward & 21,8 & 3317 & $4,710^{-2}$ \\
\hline & & leeward & 12,7 & 7330 & $6,010^{-2}$ \\
\hline
\end{tabular}

$\Delta \times 2$ can be taken arbitrarily depending on the thickness of the second layer.

The result of the concrete $\mathrm{c} 3$ represents that of the average value of the set $\mathrm{c} 1, \mathrm{c} 2$ and $\mathrm{c} 3$. C5 encompasses that of $\mathrm{c} 4, \mathrm{c} 5$ and $c 6$. The result of $c 8$ concrete combines the average value of those of c7, c8 and c9, respectively.

Thermal conductivity increases with density and cement content. It should also be noted that the thermal conductivity increases when the amount of sand added to the cement is greater than the moisture content. In addition, variations in diffusivities go hand in hand with those of conductivities during the introduction of fines and sand.

The pore structure of a material plays a dominant role in the control of its thermal conductivity [25]. For hemp concrete manufactured by a projection process, Elfoidy et al. [26] have demonstrated that the conductivity can rise to $0.49 \mathrm{w}$, $\mathrm{m}-1 . \mathrm{k}-1$ for a density of $550 \mathrm{~kg} / \mathrm{m} 3$. As a result, hemp concrete is a better insulating material than pozzolan concrete.

\section{CONCLUSION}

An attempt was made to optimize space-time meshes of a Cranck-Nicholson finite difference scheme during the heat transfer study in a single-walled wall. The wall is subjected to the limit condition of Fourier as well as to a constant temperature. The single layer wall consists of pozzolan concrete.

- It has been found that the thermal characteristics of pozzolan concrete are almost similar to those of mortars used for joints and coatings.

- The dimensional parameter $M$ which is proportional to the diffusivity and to the time step, but inversely proportional to the square of the step of the space is taken equal to unity.

- Taken together, these observations stimulated further studies using other materials such as uncooked clay bricks, stabilized clay bricks, clods used as a wall by inhabitants in the countryside.

- The results of the experimental study, which we have not presented here, are similar to the average results of the analytical solution and the numerical solution. 


\section{LIST OF TABLES}

Table 1: Physical and chemical properties of Cement.

Table 2: Pozzolan used in the study.

Table3: Chemical analysis of pozzolans.

Table4: Concretes composition.

Table5: Bilayer wall equation systems.

Table6: Synthesis of results in the case of single layer wall.

Table7: Characteristic parameters for pozzolan concrete plus marble.

\section{LIST OF FIGURES}

Fig. 1: X-ray diffraction analysis.

Fig. 2: Curve shape y1 and y2.

Fig. 3: Curves showing the intersection between $\mathrm{z} 1$ and $\mathrm{z} 2$.

Fig. 4: Behavior of curve from implicit schema of finite differences in unstable case.

Fig. 5: (HT) in the Concretes c5; (a) Solutions for $\mathrm{t}=1000 \mathrm{~s}$;

(b) Percentage difference between (AS) and (NS) for $\mathrm{t}=1000 \mathrm{~s}$.

Fig.6: (HT) in the concretes c3; (a) Solutions for $\mathrm{t}=100 \mathrm{~s}$.

(b) Percentage difference between (AS) and (NS) for $\mathrm{t}=100 \mathrm{~s}$.

Fig. 7: (HT) in the concretes c8; (a) Solutions for $\mathrm{t}=1800 \mathrm{~s}$.

(b) Percentage difference between (AS) and (NS) for $\mathrm{t}=1800 \mathrm{~s}$.

Fig. 8: (HT) in the concretes $\mathrm{c} 3+\mathrm{c} 10$; (a) Solutions for $\mathrm{t}=$ 100s; (b) Percentage difference between (AS) and (NS) for $\mathrm{t}=100 \mathrm{~s}$.

Fig. 9: (HT) in the concretes $\mathrm{c} 8+\mathrm{c} 10$; (a) Solutions for $\mathrm{t}=$ 500s; (b) Percentage difference between (AS) and (NS) for $\mathrm{t}=500 \mathrm{~s}$. 